\title{
MicroRNA 141 represses nasopharyngeal carcinoma growth through inhibiting BMI1
}

\author{
FEI LIU* ${ }^{*}$ WENJUAN WANG ${ }^{*}$, SHUO LI, QIONG YANG, JING HU, NAN ZENG and CHUNSHENG GAO \\ Department of Otolaryngology, Nanshan Affiliated Hospital of Guangdong Medical College, \\ Shenzhen, Guangdong 518052, P.R. China
}

Received July 7, 2017; Accepted July 23, 2017

DOI: $10.3892 / \mathrm{ol} .2018 .9444$

\begin{abstract}
Distant metastasis is the primary cause of mortality in patients with nasopharyngeal carcinoma (NPC). Unveiling the mechanism of metastasis will aid in shedding light on the clinical therapeutic strategies in NPC treatment. The present study revealed that the expression of microRNA 141 (miR-141) was downregulated in NPC tumor cells, particularly in metastatic ones. Ectopic expression of miR-141 blocked the proliferative and invasive ability of the tumor cells in vitro, and inhibited NPC tumor growth in vivo. Mechanistic studies identified that BMI1 served as the direct target of miR-141, and overexpression of BMI1 reversed the tumor repressor effect of miR-141. Prognostic analysis revealed that this miR-141/BMI1 signaling axis correlated with the clinical stage of patients with NPC. The study of miR-141 provided novel insight into the mechanism of NPC progression, which was correlated with the stage and metastatic state of patients. This miR-141/BMI1 axis may serve as a novel pharmacological target in NPC treatment.
\end{abstract}

\section{Introduction}

Nasopharyngeal carcinoma (NPC) is a head and neck cancer with a relatively high rate of malignancy. The tumor cells in NPC are derived from nasopharynx epithelium, and invade the surrounding tissues with the tumor growth. Facilitated by the progress of radiotherapy, the curative effect on NPC has increased. However, there are certain patients with advanced

Correspondence to: Dr Chunsheng Gao, Department of Otolaryngology, Nanshan Affiliated Hospital of Guangdong Medical College, 89 Tao Yuan Road, Shenzhen, Guangdong 518052, P.R. China

E-mail: szgaocs@qq.com

*Contributed equally

Abbreviations: NPC, nasopharyngeal carcinoma; miRNA, microRNA

Key words: nasopharyngeal carcinoma, microRNA-141, BMI1, metastasis stages of disease with a poor prognosis, owing to recurrence or distant metastasis $(1,2)$. Therefore, understanding the molecular mechanisms governing NPC tumor cells proliferation and metastasis will aid in identifying novel and more effective therapeutic methods.

MicroRNA (miRNA), which is widely expressed in numerous organisms, is a small non-coding RNA that is 19-25 nt in length. Usually, miRNA takes part in the post-transcriptional regulation of gene expression through base-pairing 3'-untranslated region (UTR) of the target mRNAs (3-5). The important regulatory role of miRNAs during biological processes have been revealed in recent decades. Recent studies also demonstrated that certain miRNAs were aberrantly expressed in different types of cancer, and served critical roles in carcinogenesis (6-8), indicating that miRNAs can serve clinically as an important indicator of cancer developmental stage. Furthermore, manipulating miRNA expression was an effective therapeutic strategy for cancer treatment (9-12). miR-141 was first identified to function in prostate cancer (13). Subsequent studies also revealed that it served critical roles in gastric and ovarian cancer $(14,15)$. However, no previous studies has focused on miR-141 in NPC.

The present study investigated the expression and function of miR-141 in NPC, and revealed its significance in early diagnosis and clinical treatment. It was demonstrated that miR-141 was a tumor repressor and was downregulated in the NPC biopsy samples. Functional studies revealed that ectopic expression of miR-141 inhibited NPC tumor cell proliferation and invasion. Further analysis revealed that BMI1 served as the direct target of miR-141. This miR-141/BMI1 signaling cascade provided a novel therapeutic strategy for NPC treatment.

\section{Materials and methods}

Ethics statement. The Research Ethics Committee of the Nanshan Affiliated Hospital of Guangdong Medical College provided ethical approval for the present study, and all patients provided written informed consent. All specimens were handled and stored anonymously according to ethical and legal standards.

Patients and specimens. Tumor samples were obtained from patients (average age of 51 years, ranging from 22 to 74 years 
old, consisting of 38 males and 13 females) with pathologically confirmed NPC (metastatic, $n=10$; non-metastatic, $n=41$ ) or nasopharyngeal mucosa chronic inflammation $(n=4)$ at the Department of Otorhinolaryngology, Nanshan Affiliated Hospital of Guangdong Medical College between June 2015 and August 2016. No patient had undergone any antitumor therapy prior to sampling. All patients were staged according to the 7th edition of the American Joint Committee on Cancer Staging Manual (16).

Cell lines. The human immortalized nasopharyngeal epithelial NP69 cell line was maintained in Keratinocyte/serum-free medium (Invitrogen; Thermo Fisher Scientific, Inc., Waltham, MA, USA) with bovine pituitary extract (BD Biosciences, Franklin Lakes, NJ, USA). Human NPC 6-10B, C666, 5-8F and SUNE1 cell lines were cultured in Dulbecco's modified Eagle's medium (Invitrogen; Thermo Fisher Scientific, Inc.) with $10 \%$ fetal bovine serum (FBS; Gibco; Thermo Fisher Scientific, Inc.). For transfection, the cells were seeded into 6-well plates the day before transfection using Lipofectamine 2000 (Invitrogen; Thermo Fisher Scientific, Inc.). 150 pM (pmol/l) miR-ctrl (5'-GGUCUGGGUAGAUCACAAUCU AC-3') or miR-141 oligo mimics (5'-UAACACUGUCUG GUAAAGAUGG-3') was transfected per well. BMI1 coding sequence was cloned into pcDNA3.1 plasmid. $2.5 \mu \mathrm{g}$ plasmids were transfected in each well. Approximately $36 \mathrm{~h}$ later, the cells were harvested for the subsequent experiments.

RNA isolation and reverse transcription-quantitative polymerase chain reaction $(q P C R)$. Total RNA was isolated from NPC cell lines using an EasyPure RNA kit (Beijing Transgen Biotech Co., Ltd., Beijing, China), according to the manufacturer's protocol. RNA $(2 \mu \mathrm{g})$ was reverse transcribed at $42^{\circ} \mathrm{C}$ for $1 \mathrm{~h}$ using $\mathrm{M}-\mathrm{MLV}$ reverse transcriptase reagents (Promega Corporation, Madison, WI, USA). For the qPCR assay, complementary DNA (cDNA) was PCR-amplified using a GoTaq qPCR Master mix (Promega Corporation) in the Light Cycler 480 II PCR system (Roche Diagnostics $\mathrm{GmbH}$, Basel, Switzerland). GAPDH was used as the internal control. The sense and anti-sense primers of miR-141 were 5'-TGGGTCCATCTTCCAGTA-3' and 5'-GGGAGCCATCTT TACCAG-3', respectively. The GAPDH sense and anti-sense primers were 5'-GAAGGTGAAGGTCGGAGT-3' and 5'-GAA GATGGTGATGGGATTTC-3', respectively. The thermocycling condition of qPCR: initial denaturation $95^{\circ} \mathrm{C} 30 \mathrm{sec}$, followed by 45 cycles: $95^{\circ} \mathrm{C} 15 \mathrm{sec}, 65^{\circ} \mathrm{C} 1 \mathrm{~min}$. Relative gene expression was presented as the comparative threshold cycle $\left(2^{-\Delta \Delta C q}\right)$ values (17) and was representative of at least three independent experiments.

Western blotting. Protein was extracted using a protein extraction kit (KGP250-2100; Nanjing KeyGen Biotech Co., Ltd., Nanjing, China) according to the manufacturer's protocol. After examining the concentration by BCA method, $10 \mu \mathrm{g}$ protein from samples were treated with Dual Color Protein Loading Buffer (Thermo Fisher Scientific, Inc.) containing reducing agent at $100^{\circ} \mathrm{C}$ for $5 \mathrm{~min}$ respectively, resolved on $10 \%$ Tris- $\mathrm{HCl}$ polyacrylamide gels, and transferred to a polyvinylidene fluoride membrane. Then the membrane was blocked by $5 \%$ skim milk for $1 \mathrm{~h}$ at room temperature. Overnight incubation $\left(4^{\circ} \mathrm{C}\right)$ with primary antibodies against the following: BMI1 (dilution, 1:1,000; catalog no. ab14389; Abcam, Cambridge, UK) and GAPDH (dilution, 1:2,000; catalog no. ab8245; Abcam) was followed by incubation $\left(37^{\circ} \mathrm{C}\right)$ with horseradish peroxidase (HRP)-conjugated anti-rabbit (dilution, 1:1,000; catalog no. NBP2-30348H; Novus Biologicals, LLC, Littleton, CO, USA) or anti-mouse (dilution, 1:1,000; catalog no. NBP2-30347H; Novus Biologicals) secondary antibodies. Immobilon Western Chemiluminescent HRP Substrate (EMD Millipore, Billerica, MA, USA) and a Tanon 5200 Luminescent Imaging Workstation (Tanon Science \& Technology Co., Ltd., Shanghai, China) were subsequently used.

Mice tumor model. Mice are raised in individually ventilated cages (IVF) in the mouse room with temperature of $18-23^{\circ} \mathrm{C}$ and 40-60\% humidity. A 12 light/12 dark cycle is used. Water and food are accessible at all times. $10 \mathrm{BALB} / \mathrm{c}$ nude male mice (Biocytogen, Worcester, MA, USA) aged 4 weeks (18-22 g body weight) were used for the tumor growth experiments. SUNE1 cells transfected with miR-141 or scrambled control were resuspended in PBS, and $1 \times 10^{6}$ cells were subcutaneously injected into the dorsal flank of the nude mice. Mice were observed and the tumor sizes were measured every two days. A total of 3 weeks later, the mice were sacrificed and the tumors were dissected and compared. For H\&E staining, the tumor tissues were fixed by $4 \%$ paraformaldehyde at $4^{\circ} \mathrm{C}$ overnight, and embedded in paraffin. Next, the tissues were sectioned into 5- $\mu \mathrm{m}$ tissues for $\mathrm{H} \& \mathrm{E}$ staining. For metastasis analysis, $2 \times 10^{6}$ SUNE1 cells diluted in $200 \mu \mathrm{l}$ PBS were injected into 10 nude mice via the tail vein. Mice were observed every two days. A total of 8 weeks later, mice were sacrificed and their lungs were dissected. The general humane endpoints for mice that required euthanasia are as follows: $20 \%$ decrease in normal body weight, the longest diameter of the tumor exceeded $20 \mathrm{~mm}$, the inability to reach food or water for more that $24 \mathrm{~h}$. All mice were euthanized by carbon dioxide with $10-30 \%$ chamber volume per minute. Animal feeding and research procedures were approved by the Animal Care and Use Ethic Committee of Nanshan Affiliated Hospital of Guangdong Medical College (Shenzhen, China).

Luciferase reporter assay. The wild-type and mutated 3'-UTR of miR-141 were cloned into firefly luciferase-expressing vector psiCHECK ${ }^{\mathrm{TM}}$ (Promega Corporation). For the luciferase reporter assay, NPC SUNE1 and 5-8F cells were co-transfected with miR-141 and BMI1 wt or mutated 3'-UTR reporter vectors by Lipofectamine 2000 (Invitrogen; Thermo Fisher Scientific, Inc.). $36 \mathrm{~h}$ later, the luciferase activity was examined using a Dual-Luciferase ${ }^{\circledR}$ Reporter assay system (catalog no. E1910; Promega Corporation), and the reporter activity were normalized by comparing with Renilla luciferase activity.

MTT and colony formation assay. For the MTT assay, NPC SUNE1 and 5-8F cells transfected with miR-141 or scrambled control were counted and 1,500 of them were seeded into 96-well plates. DMSO was used to dissolve the formazan in each well. From day 1 to day 5, the absorbance of the cells was determined at $490 \mathrm{~nm}$ using a spectrophotometric plate 

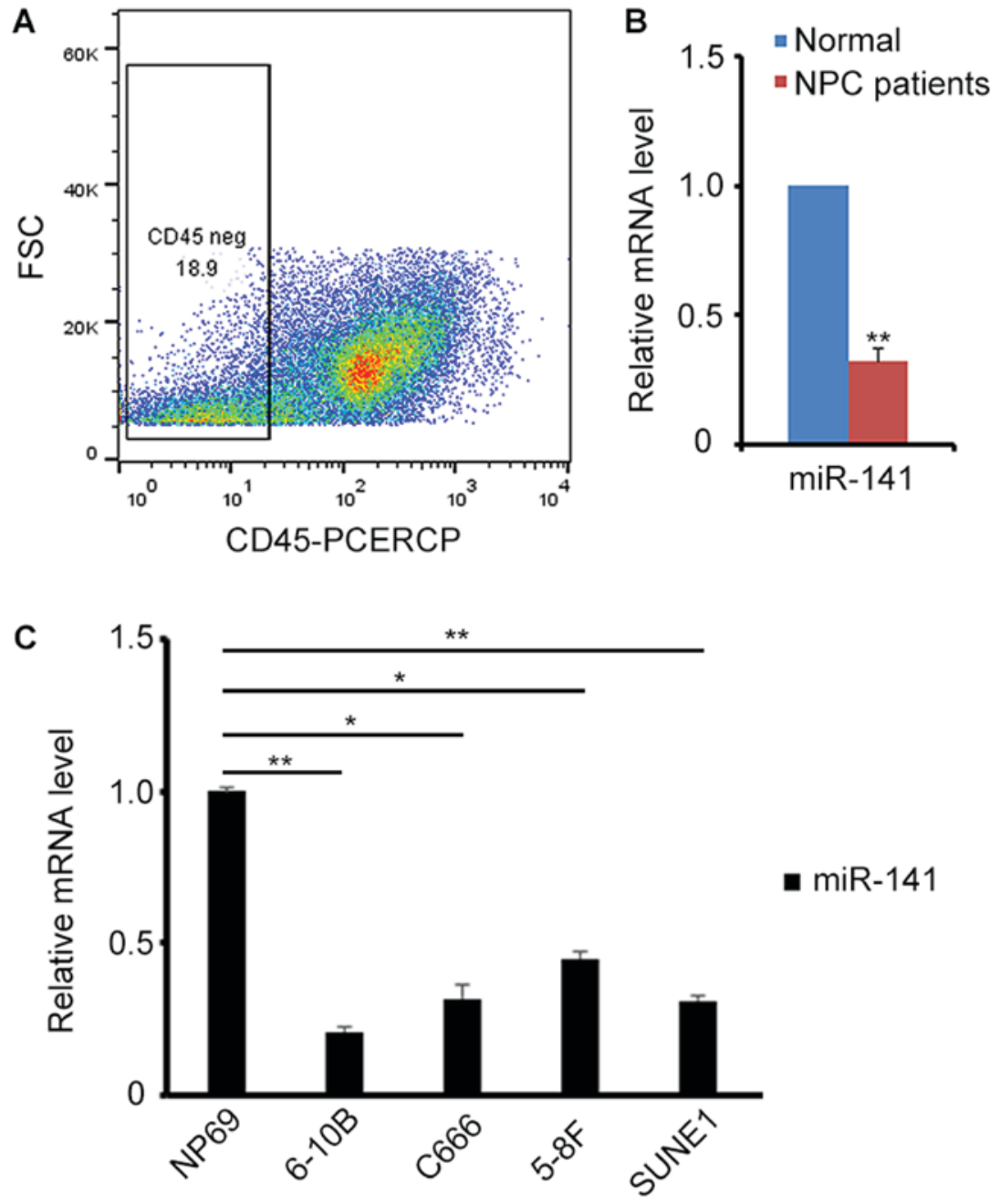

Figure 1. The expression of miR-141 is downregulated in NPC cells in vivo and in vitro. (A) FACS-sorted CD45- cells from NPC biopsy samples. (B) Expression of miR-141 in sorted NPC cells. (C) Quantitative polymerase chain reaction detection of miR-141 expression in NPC cell lines. Data are presented as the mean \pm standard deviation. P-values were calculated relative to NP69 by analysis of variance with Bonferroni's correction. ${ }^{*} \mathrm{P}<0.05,{ }^{* * *} \mathrm{P}<0.01$. miR, microRNA; NPC, nasopharyngeal carcinoma; $\mathrm{CD}$, cluster of differentiation.

reader. For the colony formation assay, 500 cells transfected with miR-141 or scrambled control were seeded into 6-well plates. The colonies were observed and counted 5-7 days later.

Cell invasion assay. A total of $5 \times 10^{4} 5-8 \mathrm{~F}$ or SUNE1 cells following transfection with miR-141 or scrambled control were resuspended in serum-free medium (DMEM, Thermo Fisher Scientific, Inc.). The Transwell chambers (Corning Incorporated, Corning, NY, USA) were placed on the upper surface of the 24-well plate, and the cells were seeded into the upper chamber. The medium supplemented with $10 \%$ FBS was placed in the lower chambers. After 10-16 h of culture, the chambers were collected and the cells on the lower surface of the chambers were fixed by absolute methanol for $20 \mathrm{~min}$ at room temperature and stained by $0.1 \%$ crystal violet for $30 \mathrm{~min}$ at room temperature. The cells on the chambers were captured at x100 magnification by an inverted microscope.

Statistical analysis. All data are presented as the mean \pm standard deviation. $\chi^{2}$ test, Fisher's exact test, analysis of variance with Bonferroni's correction and Student's t-test were used for comparisons between groups. Spearman's correlation analysis was used to examine the correlation between gene expression and disease staging. The prognostic factors were examined by univariate and multivariate analyses using the Cox proportional hazards model. $\mathrm{P}<0.05$ was considered to indicate a statistically significant difference. All statistical analyses were performed using SPSS version 18.0 (SPSS, Inc., Chicago, IL, USA).

\section{Results}

miR-141 serves as a tumor repressor in NPC. To begin with, the endogenous expression of miR-141 in NPC biopsy samples was detected. To exclude the interference of lymphocytes in these samples, the CD45 cells were sorted for the subsequent experiments by flow cytometry (Fig. 1A). A total of 23 patients were examined. Compared with healthy people, the biopsy samples from patients with NPC exhibited relatively low expression of miR-141 (Fig. 1B). Next, endogenous miR-141 expression was detected in different NPC cell lines. Consistent with the results in biopsy samples, miR-141 
A

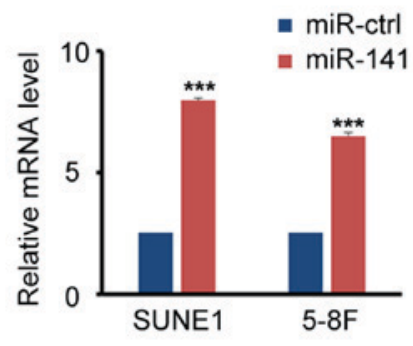

C

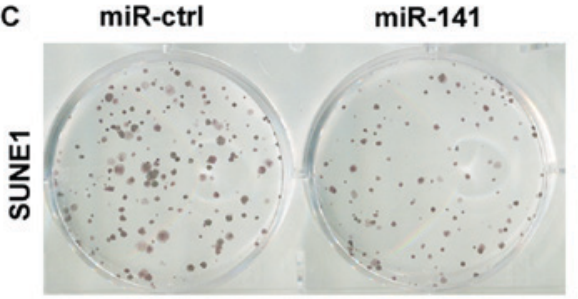

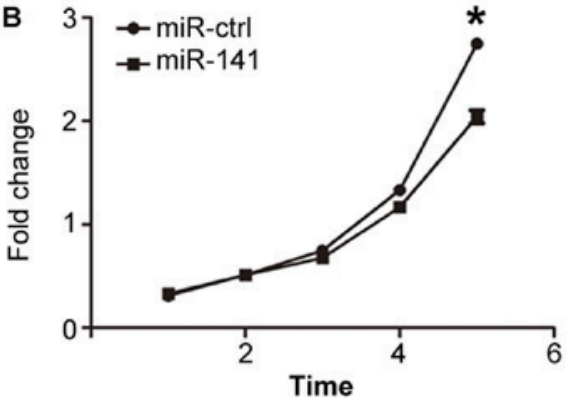

D

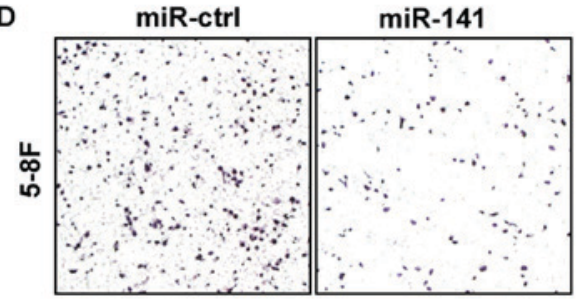

Figure 2. Overexpression of miR-141 inhibits NPC cell proliferation and metastasis. (A) Quantitative polymerase chain reaction detection of miR-141 expression following treatment with miR-141 mimics. (B) MTT assay of 5-8F cells with miR-141 overexpression. P-values were calculated by comparing with control groups at each time point using Student's t-test. (C) Plating assay of SUNE1 cells with miR-141-overexpression. (D) Transwell assay of 5-8F cells with miR-141-overexpression. Data are presented as the mean \pm standard deviation. ${ }^{*} \mathrm{P}<0.05,{ }^{* * * *} \mathrm{P}<0.001$ (Student's t-test). miR, microRNA; NPC, nasopharyngeal carcinoma; ctrl, control.

A

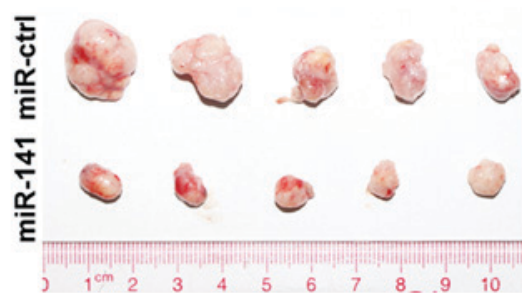

C

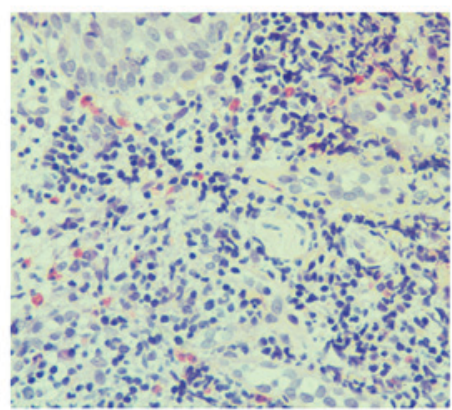

B
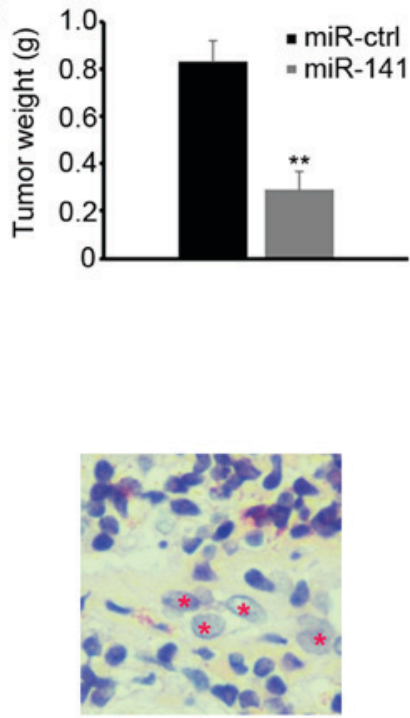

D

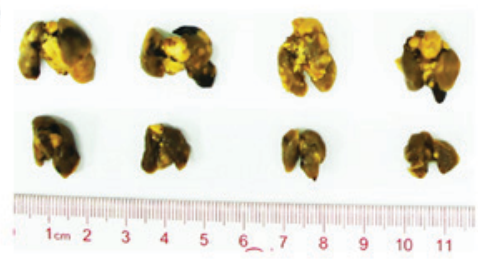

E

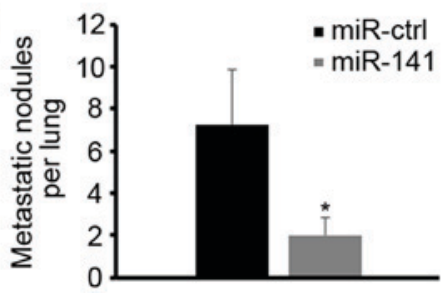

Figure 3. Ectopic expression of miR-141 suppresses NPC tumor growth in vivo. (A) SUNE1 cells with ectopic expression of miR-141 were injected into nude mice. A total of 3 weeks later, mice were sacrificed and tumor cells were dissected and observed. (B) The tumor cells were subsequently weighed. Data are presented as the mean \pm standard deviation. ${ }^{* *} \mathrm{P}<0.01$ (Student's $\mathrm{t}$-test). (C) H\&E staining of tumor tissues dissected from mice (magnification, $\mathrm{x} 400$ ). The boxed region shows a higher magnified view of the image. Red asterisks indicate the tumor cells. The lungs from mice treated with (D) miR-ctrl or (E) miR-141, respectively. The metastatic nodules on the lung surface from each mouse were counted. Data are presented as the mean \pm standard deviation. "P $<0.05$ (Student's t-test). miR, microRNA; NPC, nasopharyngeal carcinoma; ctrl, control.

expression was revealed to be decreased in NPC cell lines (Fig. 1C), implying that miR-141 may act as a tumor repressor in NPC.
To examine the role of miR-141 in NPC, the miR-141 oligo-mimics were designed to simulate the ectopic expression of miR-141 in vitro. Following transfection of miR-141 
A

Bmi1 WT 3'UTR 5'...GAAGACUUUUUCUCUGUGUUA...3' miR-141 3'...GGUAGAAAUGGUCUGUCACAAU...5'

Bmi1 MU 3'UTR 5'...GAAGACUUUUUCUCUCACAAU....3'
B

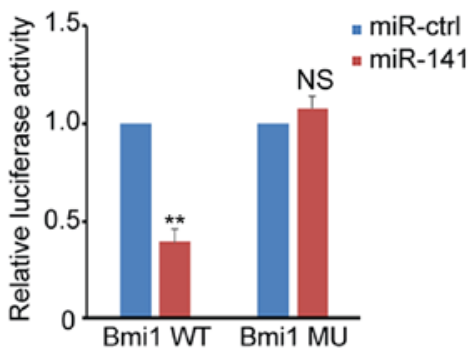

C

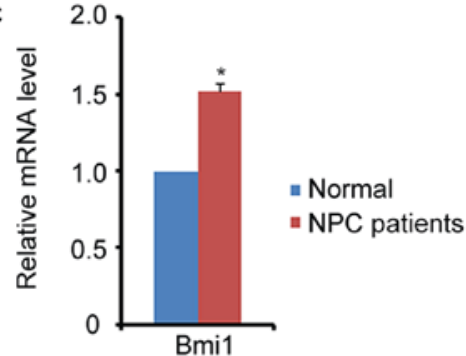

E

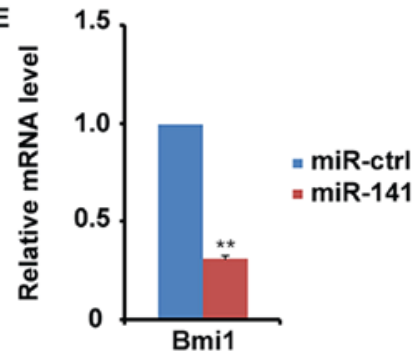

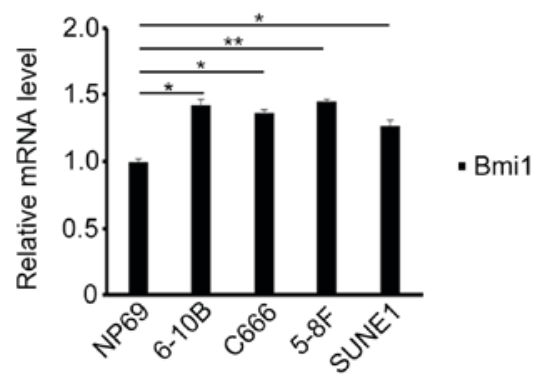

F

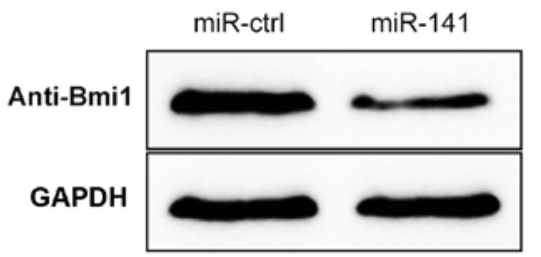

G

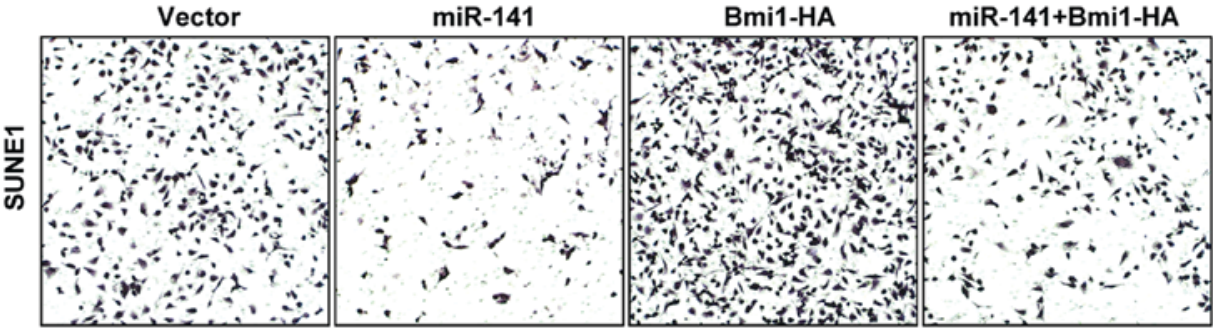

H
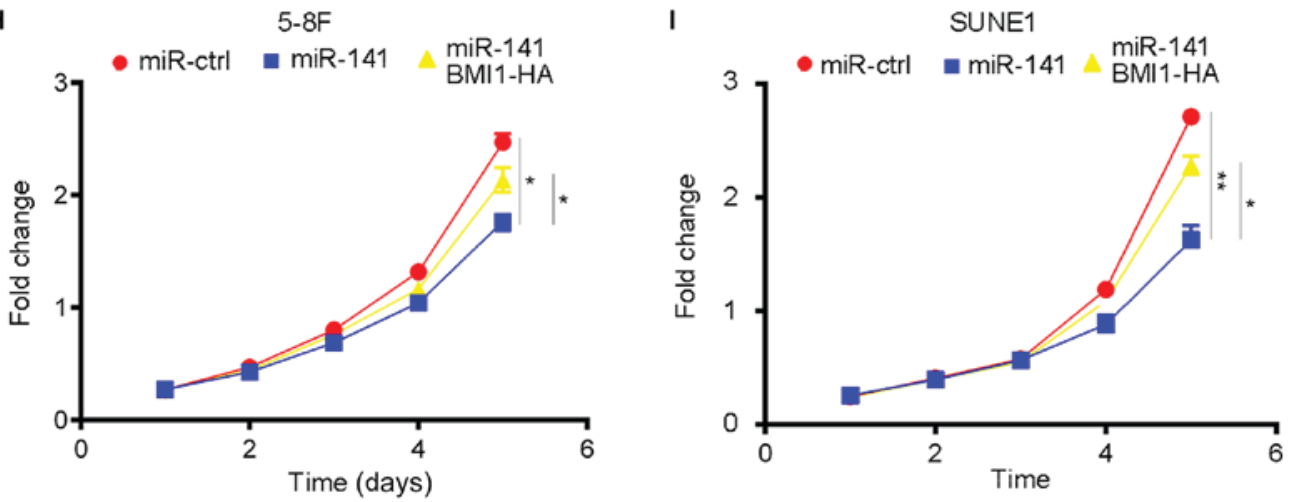

Figure 4. BMI1 served as the direct target of miR-141 in NPC cells. (A) Wild-type or mutant miR-141 target sequence of BMI1 3'-UTR. (B) Relative luciferase activity of SUNE1 cells following co-transfection with wild-type or mutant BMI1 3'-UTR reporter genes and miR-141 mimics (Student's t-test). (C) Expression of BMI1 in NPC biopsy samples (Student's t-test). (D) BMI1 expression in NPC cell lines. P-values were calculated by comparing with NP69 using ANOVA with Bonferroni's correction. (E) Quantitative polymerase chain reaction detection of BMI1 expression in SUNE1 cells with miR-141 overexpression (Student's t-test). (F) BMI1 expression in 5-8F cells with miR-141 overexpression by western blot analysis. (G) Transwell assay of SUNE1 cells with miR-141 or BMI1 overexpression. MTT assay of (H) 5-8F or (I) SUNE1 cells with miR-141-overexpression or combined with BMI1 ectopic expression. P-values were calculated by comparing with the miR-141/BMI1 group at each time point using ANOVA with Bonferroni's correction. Data are presented as the mean \pm standard deviation. ${ }^{*} \mathrm{P}<0.05,{ }^{* *} \mathrm{P}<0.01$. miR, microRNA; NPC, nasopharyngeal carcinoma; ctrl, control; UTR, untranslated region; ANOVA, analysis of variance.

mimics into NPC SUNE1 and 5-8F cells, increased expression of miR-141 was detected (Fig. 2A). To identify whether
miR-141 affects NPC cell viability, MTT and colony formation assays were performed. It was revealed that miR-141 
Table I. Correlation between miR-141 expression and clinicopathological characteristics of nasopharyngeal carcinoma.

Expression of miR-141

\begin{tabular}{|c|c|c|c|c|}
\hline Characteristic & No. patients & Low & High & $\mathrm{P}$-value \\
\hline Age, years & & & & 0.96 \\
\hline$\leq 45$ & 22 & 10 & 12 & \\
\hline$>45$ & 29 & 13 & 16 & \\
\hline Sex & & & & 0.67 \\
\hline Male & 39 & 19 & 20 & \\
\hline Female & 12 & 5 & 7 & \\
\hline VCA-IgA & & & & 0.74 \\
\hline$<1: 80$ & 11 & 6 & 5 & \\
\hline$\geq 1: 80$ & 40 & 24 & 16 & \\
\hline EA-IgA & & & & 0.54 \\
\hline$<1: 10$ & 14 & 7 & 7 & \\
\hline$\geq 1: 10$ & 37 & 15 & 22 & \\
\hline TNM stage & & & & 0.04 \\
\hline I-II & 22 & 10 & 12 & \\
\hline III-IV & 27 & 20 & 7 & \\
\hline Metastasis & & & & 0.03 \\
\hline Yes & 10 & 9 & 1 & \\
\hline No & 41 & 22 & 19 & \\
\hline
\end{tabular}

miR, microRNA; VCA-IgA, viral capsid antigen immunoglobulin A; EA-IgA, early antigen immunoglobulin A; TNM, Tumor-Node-Metastasis . P-values were analyzed by Student's t-test or Fisher's exact test, as appropriate.
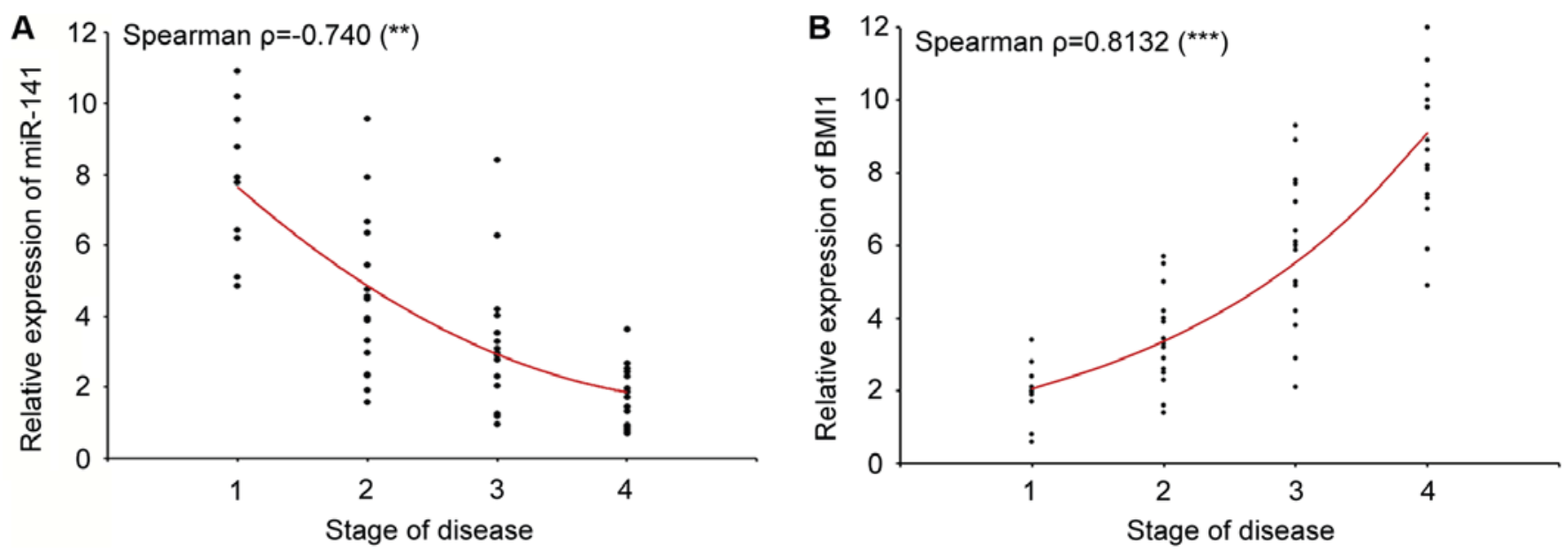

Figure 5. miR-141/BMI1 signaling axis was correlated with the disease stage of patients with NPC. (A) miR-141 expression in patients with different stages of NPC ( $\mathrm{n}=51)$. (B) Quantitative polymerase chain reaction detection of BMI1 in patients with different stages of NPC ( $\mathrm{n}=51)$. Spearman's correlation analysis was performed to analyze the correlation between mRNA expression and the disease stage of NPC. ${ }^{* *} \mathrm{P}<0.01,{ }^{* * * *} \mathrm{P}<0.001$. miR, microRNA; NPC, nasopharyngeal carcinoma.

overexpression blocked tumor cells proliferation compared with their control counterparts (Fig. 2B). The colony formation assay also revealed that ectopic expression of miR-141 inhibited tumor cell growth (Fig. 2C). To determine whether miR-141 influenced NPC cell invasive ability, a Transwell assay was performed. The results revealed that overexpression of miR-141 decreased the number of invaded tumor cells (Fig. 2D).

Next, it was determined whether ectopic expression of miR-141 affects tumor growth in vivo. SUNE1 cells transfected with miR-141 mimics or scrambled control were subcutaneously injected into the dorsal flank of nude mice $(n=10)$. A 
Table II. Univariable and multivariable analyses of prognostic parameters in patients with nasopharyngeal carcinoma.

\begin{tabular}{lcr}
\hline Variable & HR $(95 \%$ CI $)$ & P-value \\
\hline Univariate analysis & & \\
Age, $\leq 45$ years/>45 years & $1.212(0.932-2.819)$ & 0.082 \\
Sex, male/female & $1.878(1.241-3.752)$ & 0.241 \\
VCA-IgA, $<1: 80 / \geq 1: 80$ & $2.131(1.381-3.543)$ & 0.080 \\
EA-IgA, $<1: 10 / \geq 1: 10$ & $1.772(1.373-2.949)$ & $0.028^{\text {a }}$ \\
TNM stage, I-II/III-IV & $2.308(1.365-3.658)$ & $0.014^{\text {a }}$ \\
miR-141 expression, low/high & $0.525(0.035-1.384)$ & \\
Multivariate analysis & & $0.021^{\mathrm{a}}$ \\
TNM stage, I-II/III-IV & $2.015(1.103-3.223)$ & $0.018^{\mathrm{a}}$ \\
miR-141 expression, low/high & $0.423(0.103-1.186)$ & \\
\hline
\end{tabular}

${ }^{a} \mathrm{P}<0.05$. Univariate analysis, Cox proportional hazards regression model; multivariate analysis, Cox proportional hazards regression model. Variables were adopted by univariate analysis. HR, hazard ratio; CI, confidence interval; miR, microRNA; VCA-IgA, viral capsid antigen immunoglobulin A; EA-IgA, early antigen immunoglobulin A; TNM, Tumor-Node-Metastasis.

total of 3 weeks later, the mice were sacrificed and the tumors were dissected. Compared with the control, miR-141-overexpression significantly inhibited tumor growth in vivo (Fig. 3A and $\mathrm{B}) . \mathrm{H} \& \mathrm{E}$ staining confirmed that the dissected tissues were derived from NPC tumor cells (Fig. 3C). To evaluate the inhibitory effect of miR-141 on metastasis in vivo, the SUNE1 cells were injected into nude mice via the tail vein. A total of 8 weeks later, the mice were sacrificed and their lungs were dissected. Compared with the control, ectopic expression of miR-141 effectively repressed the metastasis of NPC cells in vivo (Fig. 3D and $\mathrm{E}$ ).

BMI1 functions as the direct target of miR-141 in NPC. To investigate the mechanism of miR-141 in repressing NPC growth, the present study aimed to identify the direct downstream target of miR-141. BMI1 is known as an important regulator in NPC (18). A previous study revealed that miR-141 represses BMI1 expression in diploid fibroblasts (19). Therefore, we hypothesized that BMI1 may serve as a potential miR-141 target. To verify this, BMI1 3'-UTR target sequences with and without mutations were constructed into the luciferase reporter vector (Fig. 4A). Next, the luciferase reporter assay was performed, and the results confirmed that BMI1 served as the direct target of miR-141 (Fig. 4B).

Since miR-141 is a NPC tumor repressor gene, it was investigated whether miR-141 functioned through inhibiting BMI1 expression. BMI1 expression was first detected in NPC biopsy samples and cell lines, and it was revealed that BMI1 was highly activated (Fig. 4C and D). Next, BMI1 expression was examined in SUNE1 cells with ectopic miR-141 expression, and the results demonstrated that Bmil was inhibited accordingly (Fig. 4E and F). Furthermore, overexpressing BMI1 partially rescued the inhibitory effect of NPC cells with ectopic miR-141 expression using Transwell and MTT assays (Fig. 4G-I), indicating that BMI1 functions downstream of miR-141.

The expression of miR-141 and BMII correlate with the staging status of patients with NPC. To evaluate the clinical significance of miR-141 and BMI1, their expression level was detected in 51 NPC biopsy samples. As demonstrated in Table I, it was demonstrated that miR-141 expression was correlated with the clinical stage of patients with NPC $(\mathrm{P}=0.03$, Table I), and univariable and multivariable analyses confirmed this correlation (Table II). Patients with advanced stages of disease or metastasis exhibited relatively lower expression of miR-141 than patients with earlier stages of disease (Fig. 5A). Furthermore, patients with low expression of miR-141 had high expression of BMI1 (Fig. 5A and B), indicative of the regulatory role of miR-141 and BMI1 in vivo.

\section{Discussion}

The present study revealed that miR-141 was downregulated in NPC clinical samples and cell lines. Ectopic expression of miR-141 blocked tumor cell proliferation and invasion. Mechanistic analysis identified that BMI1 served as the direct target of miR-141, and overexpressing BMI1 partially rescued the tumor suppressive phenotype of miR-141. Furthermore, the miR-141/BMI1 signaling cascade was correlated with NPC clinical staging. Patients with metastases have relatively lower expression levels of miR-141 and higher expression levels of BMI1, which suggested that ectopic expression of BMI1 facilitated NPC tumor cell metastasis in vivo.

Tumor cell growth requires a series of gene transcription and modification networks. Understanding these regulatory networks may aid in identifying the mechanism of tumor cell initiation and growth, so as to develop precise medical strategies for clinical use. Previous studies have demonstrated that several miRNAs were aberrantly expressed and functioned in NPC tumor cells, as well as being involved in cell proliferation, migration and metabolism processes $(6,7,9)$. Furthermore, recent studies have demonstrated that circulating free nucleic acid (cfNA) in patients with cancer were more highly concentrated (20-22). Detecting circulating miRNAs has been emerging as a novel early diagnosis strategy to identify the clinical stage of patients with cancer $(22,23)$. Therefore, it 
would be of great importance to study the whole miRNAs regulation network during NPC development.

Recent studies have revealed that miR-141 was active in prostate cancer and ovarian cancer $(14,15)$, and served an inhibitory role in gastric cancer growth (13). Several miRNAs were reported to serve roles in manipulating NPC development (24-30). However, the expression and function of miR-141 in NPC has not been identified. The results of the present study established the tumor repressor effect of miR-141 in NPC, and confirmed that miR-141 functioned through regulating its target, BMI1. Previous results confirmed that BMI1 was an important oncogene in NPC. Inhibiting BMI1 was regarded as the effective method to block NPC proliferation and invasion $(16,31,32)$. The results provided a direct BMI1 regulator in NPC, which may aid in shedding light on the clinical application of pharmacological inhibition of BMI.

\section{Acknowledgements}

The authors would like to thank Dr Lei Wang from the Central Laboratory and Dr Wenchao Yu from the Clinical Laboratory of Nanshan Affiliated Hospital of Guangdong Medical College for their help in data analysis.

\section{Funding}

The present study was supported by a grant from the Knowledge Innovation Project of Shenzhen (grant no. JCYJ20150402152130190). The funder did not participate in study design, data analysis and manuscript publication.

\section{Authors' contributions}

FL and CG conceived the project. FL and WW carried out most of the experiments. SL, QY, JH, and NZ participated in data analysis and interpretation of results. All authors read and approved the manuscript.

\section{Availability of data and materials}

All data generated or analyzed during this study are included in this published article.

\section{Ethics approval and consent to participate}

This study was performed in accordance with the ethical standards and according to the Declaration of Helsinki and according to national and international guidelines and has been approved by the ethics committee of Nanshan Affiliated Hospital of Guangdong Medical College. Patients provided written informed consent.

\section{Patient consent for publication}

All patients have provided written informed consent for the publication of this manuscript and any associated images.

\section{Competing interests}

The authors declare that they have no competing interests.

\section{References}

1. Lai SZ, Li WF, Chen L, Luo W, Chen YY, Liu LZ, Sun Y, Lin AH, Liu MZ and Ma J: How does intensity-modulated radiotherapy versus conventional two-dimensional radiotherapy influence the treatment results in nasopharyngeal carcinoma patients? Int J Radiat Oncol Biol Phys 80: 661-668, 2011.

2. Chen Y, Sun Y, Liang SB, Zong JF, Li WF, Chen M, Chen L, Mao YP, Tang LL, Guo Y, et al: Progress report of a randomized trial comparing long-term survival and late toxicity of concurrent chemoradiotherapy with adjuvant chemotherapy versus radiotherapy alone in patients with stage III to IVB nasopharyngeal carcinoma from endemic regions of China. Cancer 119: 2230-2238, 2013.

3. Plaisance-Bonstaff $\mathrm{K}$ and Renne R: Viral miRNAs. Methods Mol Biol 721: 43-66, 2011.

4. He L and Hannon GJ: MicroRNAs: Small RNAs with a big role in gene regulation. Nat Rev Genet 5: 522-531, 2004.

5. Zamore PD and Haley B: Ribo-gnome: The big world of small RNAs. Science 309: 1519-1524, 2005.

6. Calin GA and Croce CM: MicroRNA-cancer connection: The beginning of a new tale. Cancer Res 66: 7390-7394, 2006.

7. Esquela-Kerscher A and Slack FJ: Oncomirs-microRNAs with a role in cancer. Nat Rev Cancer 6: 259-269, 2006.

8. He L, Thomson JM, Hemann MT, Hernando-Monge E, Mu D, Goodson S, Powers S, Cordon-Cardo C, Lowe SW, Hannon GJ and Hammond SM: A microRNA polycistron as a potential human oncogene. Nature 435: 828-833, 2005.

9. Krützfeldt J, Rajewsky N, Braich R, Rajeev KG, Tuschl T, Manoharan M and Stoffel M: Silencing of microRNAs in vivo with 'antagomirs'. Nature 438: 685-689, 2005.

10. Elmén J, Lindow A, Silahtaroglu M, Bak M, Christensen A, Lind-Thomsen M, Hedtjärn M, Hansen HF, Hansen EM, Straarup EM, et al: Antagonism of microRNA-122 in mice by systemically administered LNA-antimiR leads to up-regulation of a large set of predicted target mRNAs in the liver. Nucleic Acids Res 36: 1153-1162, 2008.

11. Obad S, dos Santos CO, Petri A, Heidenblad M, Broom O, Ruse C, Fu C, Lindow M, Stenvang J, Straarup EM, et al: Silencing of microRNA families by seed-targeting tiny LNAs. Nat Genet 43: 371-378, 2011.

12. Elmén J, Lindow M, Schütz S, Lawrence M, Petri A, Obad S, Lindholm M, Hedtjärn M, Hansen HF, Berger U, et al: LNA-mediated microRNA silencing in non-human primates. Nature 452: 896-899, 2008.

13. Yaman Agaoglu F, Kovancilar M, Dizdar Y, Darendeliler E, Holdenrieder S, Dalay N and Gezer U: Investigation of miR-21, miR-141, and miR-221 in blood circulation of patients with prostate cancer. Tumour Biol 32: 583-588, 2011.

14. Mateescu B, Batista L, Cardon M, Gruosso T, de Feraudy Y, Mariani O, Nicolas A, Meyniel JP, Cottu P, Sastre-Garau X and Mechta-Grigoriou F: miR-141 and miR-200a act on ovarian tumorigenesis by controlling oxidative stress response. Nat Med 17: 1627-1635, 2011.

15. Zuo QF, Zhang R, Li BS, Zhao YL, Zhuang Y, Yu T, Gong L, Li S, Xiao B and Zou QM: MicroRNA-141 inhibits tumor growth and metastasis in gastric cancer by directly targeting transcriptional co-activator with PDZ-binding motif, TAZ. Cell Death Dis 6: e1623, 2015.

16. Edge SB and Compton CC: The American Joint Committee on Cancer: The 7th edition of the AJCC cancer staging manual and the future of TNM. Ann Surg Oncol 17: 1471-1474, 2010.

17. Livak KJ and Schmittgen TD: Analysis of relative gene expression data using real-time quantitative PCR and the 2(-Delta Delta C(T)) method. Methods 25: 402-408, 2001.

18. Song LB, Zeng MS, Liao WT, Zhang L, Mo HY, Liu WL, Shao JY, Wu QL, Li MZ, Xia YF, et al: Bmi-1 is a novel molecular marker of nasopharyngeal carcinoma progression and immortalizes primary human nasopharyngeal epithelial cells. Cancer Res 66: 6225-6232, 2006.

19. Dimri M, Carroll JD, Cho JH and Dimri GP: microRNA-141 regulates BMI1 expression and induces senescence in human diploid fibroblasts. Cell Cycle 12: 3537-3546, 2013.

20. Gormally E, Caboux E, Vineis P and Hainaut P: Circulating free DNA in plasma or serum as biomarker of carcinogenesis: Practical aspects and biological significance. Mutat Res 635: 105-117, 2007.

21. Schwarzenbach H, Hoon DS and Pantel K: Cell-free nucleic acids as biomarkers in cancer patients. Nat Rev Cancer 11: 426-437, 2011.

22. Kosaka N, Iguchi H and Ochiya T: Circulating microRNA in body fluid: A new potential biomarker for cancer diagnosis and prognosis. Cancer Sci 101: 2087-2092, 2010.

23. Kang K, Peng X, Luo J and Gou D: Identification of circulating miRNA biomarkers based on global quantitative real-time PCR profiling. J Anim Sci Biotechnol 3: 4, 2012. 
24. Lu J, Luo H, Liu X, Peng Y, Zhang B, Wang L, Xu X, Peng X, Li G, Tian W, et al: miR-9 targets CXCR4 and functions as a potential tumor suppressor in nasopharyngeal carcinoma. Carcinogenesis 35: 554-563, 2014.

25. Lu J, He ML, Wang L, Chen Y, Liu X, Dong Q, Chen YC, Peng Y, Yao KT, Kung HF and Li XP: MiR-26a inhibits cell growth and tumorigenesis of nasopharyngeal carcinoma through repression of EZH2. Cancer Res 71: 225-233, 2011.

26. Liu N, Tang LL, Sun Y, Cui RX, Wang HY, Huang BJ, He QM, Jiang W and Ma J: MiR-29c suppresses invasion and metastasis by targeting TIAM1 in nasopharyngeal carcinoma. Cancer Lett 329: 181-188, 2013.

27. Zhang LY, Ho-Fun Lee V, Wong AM, Kwong DL, Zhu YH, Dong SS, Kong KL, Chen J, Tsao SW, Guan XY and Fu L: MicroRNA-144 promotes cell proliferation, migration and invasion in nasopharyngeal carcinoma through repression of PTEN. Carcinogenesis 34: 454-463, 2013.

28. Xia H, Cheung WK, Sze J, Lu G, Jiang S, Yao H, Bian XW, Poon WS, Kung HF and Lin MC: miR-200a regulates epithelial-mesenchymal to stem-like transition via ZEB2 and beta-catenin signaling. J Biol Chem 285: 36995-37004, 2010.
29. Liu N, Jiang N, Guo R, Jiang W, He QM, Xu YF, Li YQ, Tang LL, Mao YP, Sun Y and Ma J: MiR-451 inhibits cell growth and invasion by targeting MIF and is associated with survival in nasopharyngeal carcinoma. Mol Cancer 12: 123, 2013.

30. Yi C, Wang Q, Wang L, Huang Y, Li L, Liu L, Zhou X, Xie G, Kang T, Wang H, et al: MiR-663, a microRNA targeting $\mathrm{p} 21$ (WAF1/CIP1), promotes the proliferation and tumorigenesis of nasopharyngeal carcinoma. Oncogene 31: 4421-4433, 2012.

31. Qin L, Zhang X, Zhang L, Feng Y, Weng GX, Li MZ, Kong QL, Qian CN, Zeng YX, Zeng MS, et al: Downregulation of BMI-1 enhances 5-fluorouracil-induced apoptosis in nasopharyngeal carcinoma cells. Biochem Biophys Res Commun 371: 531-535, 2008.

32. Alajez NM, Shi W, Hui AB, Yue S, Ng R, Lo KW, Bastianutto C, O'Sullivan B, Gullane P and Liu FF: Targeted depletion of BMI1 sensitizes tumor cells to P53-mediated apoptosis in response to radiation therapy. Cell Death Differ 16: 1469-1479, 2009.

(c) (5) (9) This work is licensed under a Creative Commons cc) Attribution 4.0 International (CC BY-NC 4.0) License 